\title{
Self-Monitoring and Self-Delivery of Self-Assembled Fluorescent Nanoparticles in Cancer Therapy
}

This article was published in the following Dove Press journal:

International Journal of Nanomedicine

\author{
Hongmei Liu ${ }^{1,2}$ \\ Minghao Yuan ${ }^{1,2}$ \\ Yushi Liu',2 \\ Yiping Guo ${ }^{3}$ \\ Haijun Xiao ${ }^{4}$ \\ Li Guo 1,2 \\ Fei Liu ${ }^{1,2}$
}

'School of Pharmacy, Chengdu University of Traditional Chinese Medicine, Chengdu, 6I II37, People's Republic of China; ${ }^{2}$ State Key Laboratory of Characteristic Chinese Medicine Resources in Southwest China, Chengdu, 6I II37, People's Republic of China; ${ }^{3}$ Quantitative and Systems Biology Program, University of California, Merced, CA, 95343, USA; ${ }^{4}$ Centre of Polymer Systems, Tomas Bata University in Zlin, Zlin, 7600I, Czech Republic
Correspondence: Li Guo

Chengdu University of Traditional

Chinese Medicine, Chengdu, 611137,

People's Republic of China

Tel +86 28-61800237

Email guoli@cdutcm.edu.cn
Purpose: Due to the shortcomings of nanocarriers, the development of carrier-free nanodelivery systems has attracted more and more attention in cancer treatment. However, there are few studies on carrier-free nanosystems that can simultaneously achieve monitoring functions. Here a multifunctional carrier-free nanosystem loaded with curcumin and irinotecan hydrochloride was established for the treatment and monitoring of gastric cancer.

Methods: In this study, an irinotecan hydrochloride-curcumin nanosystem in the early stage (the system is named SICN) was prepared. Based on the fluorescence of curcumin, flow cytometry, laser confocal microscopy, and zebrafish fluorescence imaging were used to study the monitoring function of SICN in vivo and in vitro. In addition, HGC-27 human gastric cancer cells were used to study SICN cytotoxicity.

Results: Flow cytometry and zebrafish fluorescence imaging monitoring results showed that the uptake of SICN was significantly higher than free curcumin, and the excretion rate was lower. SICN had higher accumulation and retention in cells and zebrafish. Laser confocal microscopy monitoring results showed that SICN was internalized into HGC-27 cells through multiple pathways, including macropinocytosis, caveolin, and clathrin-mediated and clathrin -independent endocytosis, and distributed intracellularly throughout the whole cytoplasm, including lysosomes and Golgi apparatus. In vitro cell experiments showed that SICN nanoparticles were more toxic than single components, and HGC-27 cells had more absorption and higher toxicity to nanoparticles under slightly acidic conditions.

Conclusion: SICN is a promising carrier-free nanoparticle, and the combination of two single-component therapies can exert a synergistic antitumor effect. When exposed to a tumor acidic environment, SICN showed stronger cytotoxicity due to charge conversion. More importantly, the nanoparticles' self-monitoring function has been developed, opening up new ideas for combined tumor therapy.

Keywords: carrier-free, irinotecan hydrochloride, curcumin, multifunctional nanoparticles

\section{Introduction}

Cancer is a major public health problem worldwide ${ }^{1}$ and second to cardiovascular disease in threatening human life. ${ }^{2}$ There has been huge progress in cancer treatment, including surgery, radiotherapy, chemotherapy, and immunotherapy. ${ }^{3-5}$ Chemotherapy remains the most common therapeutic modality due to its high efficiency. However, clinical trials have proven that chemotherapy has many problems, such as poor targeting, systemic effects, and indiscriminate destruction of normal cells, resulting in various side effects. ${ }^{6,7}$ In recent years, targeted cancer therapy based on nanoplatforms has expanded tremendously, ${ }^{8}$ which greatly enhanced targeting, ${ }^{9}$ improved efficacy, and reduced side effects. ${ }^{10}$ Nevertheless, the fabrication of targeting nanocarriers is 
complicated, and nanocarriers may also have the risk of poor stability, nondegradability, and long-term toxicity. ${ }^{11}$ Only a few nanodrugs have been approved for clinical use. ${ }^{12}$ Therefore, it is more attractive to develop a targeted, carrierfree nanodelivery system.

Nanodrugs need to overcome a series of barriers (circulation, accumulation, penetration, internalization, and release $)^{13}$ to affect solid tumors. In other words, the amount of nanomedicine that can be effectively delivered to tumors and absorbed by tumor cells is pretty small, leading to a significant reduction in bioavailability. Previous studies have shown that the use of nanoparticle therapeutic media to respond to external stimuli (such as light, ${ }^{14} \mathrm{pH},{ }^{15}$ enzymes, ${ }^{16}$ magnetic field, ${ }^{17}$ and temperature ${ }^{18}$ ) can significantly improve antitumor efficacy. Quan et al designed a nanomedicine that responds to the highly expressed $\gamma$-glutamyl transpeptidase on the tumor cell surface, which can achieve high uptake and infiltration of distal tumor cells from the blood vessels. ${ }^{13}$ Consequently, it is interesting to utilize the tumor microenvironment or tumor heterogeneity to enhance drug uptake and penetration.

Besides improving therapeutic efficacy with nanotechnology, it is also highly desirable to simultaneously achieve real-time monitoring of the uptake and release or distribution of drug molecules within the nanosystem in vivo and vitro. So far, an effective and prevalent strategy for achieving monitoring is to encapsulate chemotherapeutic drugs into fluorescent nanomaterials of inert carriers or construct nanoparticles with drugs and fluorescent markers. ${ }^{19-21}$ Nevertheless, the preparation of nanocarriers is not easy and may have potential toxicity. The low drug loading caused by carriers or fluorescent markers severely limits the application of multifunctional therapy. ${ }^{22}$ Therefore, it is necessary to utilize therapeutic and nontoxic, fluorescent small-molecule, and chemotherapeutic drugs to construct a multifunctional nanodelivery system that can simultaneously realize selfmonitoring, improve curative effects, and reduce side effects.

Given these challenges, a carrier-free drug self-delivery system was developed based on a molecular structure that is easy to construct and can respond to $\mathrm{pH}$. In our previous research, a drug self-delivery system of camptothecin derivatives and curcumin was constructed, and it has been proven to be effective against colon cancer. The severe clinical diarrhea effect of irinotecan is greatly reduced due to the change in targeting. ${ }^{23}$ However, as a fluorescent small-molecule drug, curcumin has not been fully developed for its self-monitoring function. In this study, a carrier-free, self-assembly strategy was used again to prepare multifunctional nanoparticles of curcumin and camptothecin derivatives by precipitation. Selfassembled nanoparticles can significantly improve the anticancer effect of gastric cancer cells. Meanwhile, their adjustable surface charge in a slightly acidic tumor environment greatly enhances the nanoparticles' uptake and anticancer activity. More importantly, based on the fluorescence of curcumin, nanoparticles can be used to monitor the absorption and uptake of gastric cancer cells and zebrafish in real-time, as well as to further explore the internalization and transport pathways of nanoparticles (Figure 1). In addition, curcumin has high biological safety and broad antitumor activity ${ }^{24,25}$ and can be used as a promising fluorescent marker for tumor nanotherapy research.

\section{Materials and Methods \\ Materials}

Irinotecan hydrochloride, curcumin, poloxamer 105, mannitol, and dimethyl sulfoxide (DMSO) were purchased from Sigma-Aldrich and used as received (USA). Dulbecco's modified Eagle's medium (DMEM; pH 7.5) and HQ fetal bovine serum (FBS) were purchased from TransGen Biotech Co., Ltd. (Beijing, China). Wortmannin, cytochalasin D, genistein, and methyl- $\beta$-cyclodextrin were purchased from Meilun Biotechnology Co., Ltd. (Dalian, China). Chlorpromazine was obtained from Selleck Chemicals (Shanghai, China). Hydrochloric acid was purchased from Chengdu Kelong Chemical Reagent Factory (Chengdu, China). Phosphate-buffered saline (PBS; pH 7.4) was prepared in the laboratory, and ultrapure water was produced using a Milli-Q integral water purification system.

\section{Preparation of SICN Nanoparticle}

SICN Nanoparticles was have been prepared by precipitation method in our previous paper. ${ }^{23}$ In brief, Irinotecan hydrochloride $(6.2 \mathrm{mg})$ and curcumin $(3.7 \mathrm{mg}$ ) (molar ratio 1:1) were dissolved in dimethyl sulfoxide (DMSO, $300 \mu \mathrm{L}$ ), and $0.5 \mathrm{mg}$ of non-ionic surfactant poloxamer 105 was added. The suspension was obtained by adding ultra pure water $(30 \mathrm{~mL})$ into DMSO solvent under magnetic stirring, and then the organic solvent was removed by dialysis. Nanoparticle powder was obtained after lyophilization with mannitol.

\section{Characterization of SICN NPs}

The particle size in different solvents at room temperature were measured by Nicomp 380 ZL S Laser Particle Size Analyzer (PSS, USA). Scanning electron microscopy (SEM) 


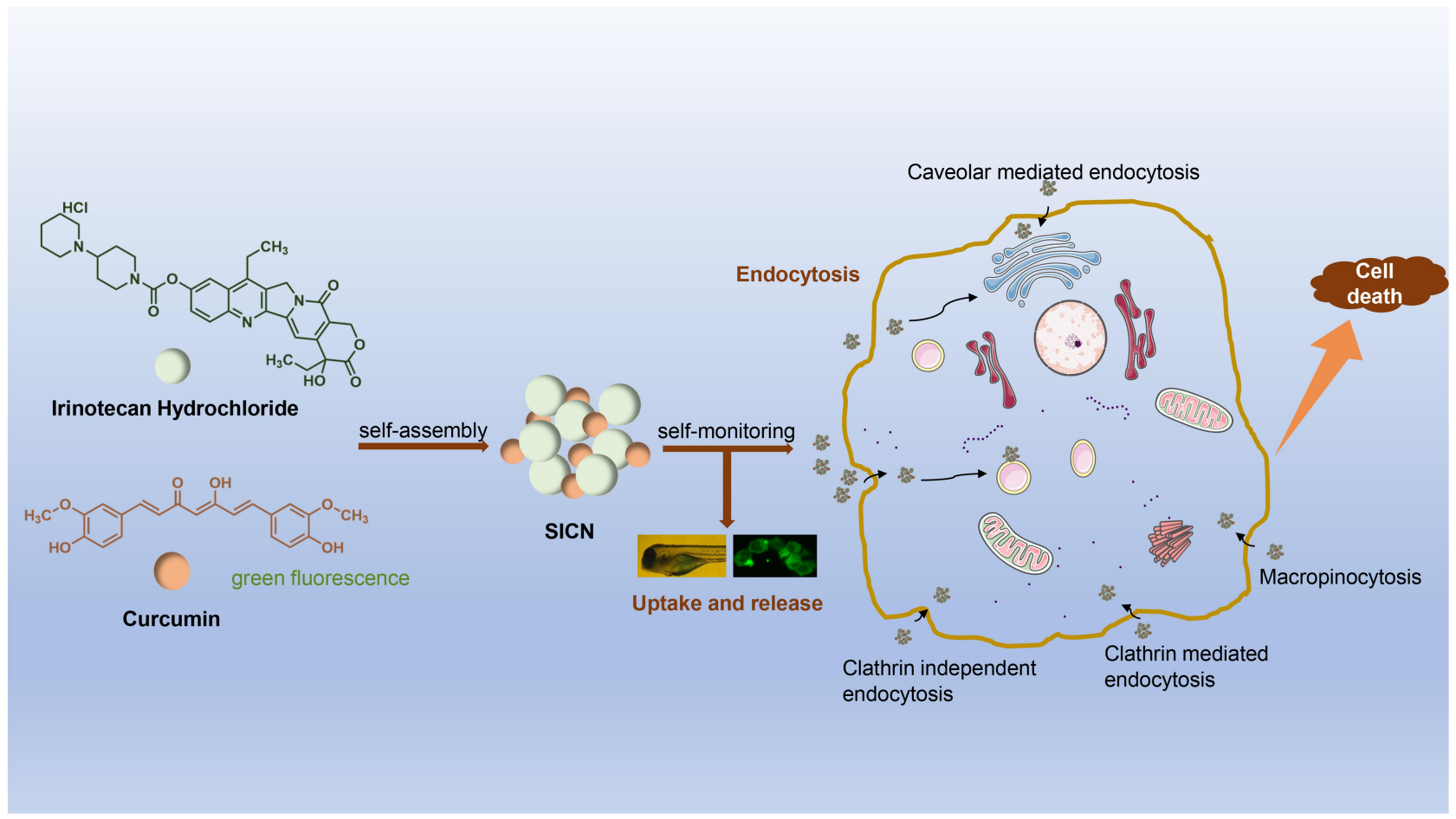

Figure I Application of self-monitoring function of self-assembled SICN nanoparticles based on the fluorescence of curcumin.

and transmission electron microscopy (TEM) were used to observe the morphology and structure of nanoparticles. The diffraction behavior of nanoparticles were characterized by a Bruker X-Ray Diffraction Analyzer (XRD, Bruker, Germany). Multifunctional microplate reader SpectraMaxi3 (Molecular Devices, America) was applied for exploring the drug release and fluorescence change.

\section{Cell Culture}

HGC-27 cell lines were purchased Procell Life Science\&Technology Co., Ltd. (No: 8200F031002) and were incubated in DMEM supplemented with 10\% TransSerum $^{\mathrm{TM}}$ HQ FBS and 1\% penicillin-streptomycin at $37^{\circ} \mathrm{C}$ at a humidified atmosphere containing $5 \% \mathrm{CO}_{2}$.

\section{In vitro Cellular Uptake and Release of SICN}

Cellular uptake and release by HGC-27 cells were performed using flow cytometry. HGC-27 cells were seeded in 12-well plates at $1 \times 10^{5}$ cells/well and treated with SICN and curcumin $(12.5 \mu \mathrm{M} / \mathrm{L})$. At designated time intervals, cells were harvested after trypsinization and centrifuged at $1500 \mathrm{rpm}$ for $5 \mathrm{~min}$. Subsequently, the cell pellets were resuspended in $1 \mathrm{~mL}$ of $1 \times$ PBS. Flow cytometry was done using a BD FACSVerse flow cytometer (BD Biosciences).

\section{Cellular Uptake and Release of SICN in Zebrafish}

AB strain zebrafish was supported by the zebrafish experimental platform at Chengdu University of Traditional Chinese Medicine (Chengdu, China), which was approved by the Ethics Review Committee of Chengdu University of Traditional Chinese Medicine (Ethics review record number:2018-16). The use of zebrafish follows Laboratory animal-Biosafety requirement laboratory animal experiment (T/CALAS7-2017) promulgated by the Chinese Society of Laboratory Animals. Zebrafish husbandry and breeding were performed according to the zebrafish book standards. ${ }^{26}$ Adult zebrafish were maintained at $28.5^{\circ} \mathrm{C}$ under a 14 $\mathrm{h}$ light/10 $\mathrm{h}$ dark cycle in fish water $(\mathrm{pH} 7.2-7.5$ and conductivity $500-550 \mu \mathrm{s} / \mathrm{cm}$ ). Zebrafish embryos were obtained from adults segregated by sex and mated in tanks overnight. Spawning was induced in the next light cycle when the light was turned on. Embryos were incubated at $28.5^{\circ} \mathrm{C}$, and larvae at 5 days postfertilization were incubated with $12.5 \mu \mathrm{M} / \mathrm{L}$ SICN and curcumin. At designated time intervals, each larva's fluorescence was 
observed, and larvae were photographed immediately with a Leica fluorescence microscope. Simultaneously, to observe SICN excretion in vivo, the medicine was replaced with fresh fish-water after $4 \mathrm{~h}$ administration and imaged.

\section{Endocytosis and Intracellular Transport of SICN Nanoparticles}

HGC-27 cells were seeded on $35 \mathrm{~mm}$ glass-bottomed culture dishes and incubated at $37^{\circ} \mathrm{C}, 5 \% \mathrm{CO}_{2}$ for $12 \mathrm{~h}$. After removing the original medium, $1 \mathrm{~mL}$ DMEM (with $1 \%$ FBS and $1 \%$ penicillin-streptomycin) containing predetermined concentrations of chlorpromazine, genistein, methyl- $\beta$ cyclodextrin, wortmannin, and cytochalasin $\mathrm{D}$ was added to the culture dishes or placed in the dishes at a $4^{\circ} \mathrm{C}$ environment. After incubation for $0.5 \mathrm{~h}$, the medium was removed, and SICN $(6.25 \mu \mathrm{M} / \mathrm{L})$ was added to each culture dish to continue incubation for $2 \mathrm{~h}$. The $4^{\circ} \mathrm{C}$ group was maintained at $4^{\circ} \mathrm{C}$. Before imaging, the culture dishes were washed thoroughly with ice-cold PBS. Finally, the fluorescent images of cells were recorded with a confocal laser scanning microscope (CLSM; Leica), and the fluorescence intensity of cells was measured at the same time.

To further study the intracellular transport pathway of SICN, HGC-27 cells were incubated with SICN for $6 \mathrm{~h}$ and then stained with LysoTracker Red and Golgi-Tracker Red (Beyotime Biotechnology). The staining process was performed according to the manufacturer's instructions. ProLong ${ }^{\text {TM }}$ Live Antifade Reagent (Thermo Fisher Scientific) was added before observation with CLSM.

\section{Cell Cytotoxicity Assay}

HGC-27 cells were seeded on 96-well plates in DMEM containing $10 \%$ FBS and 1\% penicillin-streptomycin and continued to culture for $24 \mathrm{~h}$. After removing the original medium in each well, $100 \mu \mathrm{L}$ DMEM (with 1\% FBS and $1 \%$ penicillin-streptomycin) containing predetermined concentrations of irinotecan hydrochloride, curcumin, and SICN were added to the designated wells. After a further culture for $48 \mathrm{~h}$, the original culture medium was removed before adding the mixture containing $90 \mu \mathrm{L}$ DMEM (with $1 \%$ FBS and $1 \%$ penicillin-streptomycin) and $10 \mu \mathrm{L}$ 3-(4,5-dimethylthiazol-2-yl)-2,5-diphenyltetrazolium bromide (MTT) stock solution $(5 \mathrm{mg} / \mathrm{mL}$ in PBS) and incubated for $4 \mathrm{~h}$. Then, the medium containing MTT was completely removed, followed by the addition of $150 \mu \mathrm{L}$ DMSO to each well. Cell viabilities were determined by reading the absorbance at $490 \mathrm{~nm}$ using Gen5 microplate reader and imager software (BioTek). Cells incubated with $1 \%$ serum-supplemented medium represented $100 \%$ cell survival. Six replicate wells were run for each concentration.

\section{Cell Apoptosis Assay}

Apoptosis in cells was visualized using a KeyGen Annexin V-fluorescein isothiocyanate (FITC) Apoptosis Detection Kit. HGC-27 cells were placed in a 12-well plate at a density of $1 \times 10^{5}$ cells per well and further incubated for $24 \mathrm{~h}$. Apoptosis in cells was induced by treating cells with curcumin, irinotecan hydrochloride, or SICN nanoparticles at $3.125 \mu \mathrm{M} / \mathrm{L}$ concentration for $48 \mathrm{~h}$. Negative control was prepared by incubating cells in the absence of an inducing agent. Cells were harvested, washed twice with cold PBS, and stained with KeyGen Annexin V and propidium iodide (PI) according to the manufacturer's instructions. Stained cells were analyzed by a BD FACSVerse flow cytometer.

\section{Cell Cycle Assay}

Cycle in cells was visualized using a MultiSciences Cell Cycle Staining Kit. HGC-27 cells were placed in a 12-well plate at a density of $1 \times 10^{5}$ cells per well and further incubated for $24 \mathrm{~h}$ and treated with curcumin, irinotecan hydrochloride, or SICN nanoparticles at $3.125 \mu \mathrm{M} / \mathrm{L}$ concentration for $48 \mathrm{~h}$. Negative control was prepared by incubating cells in a medium without any drugs. Cells were harvested, washed twice with cold PBS, and stained with MultiSciences Cell Cycle agents according to the manufacturer's instructions. Stained cells were analyzed by a BD FACSVerse flow cytometer.

\section{Results and Discussion Characterization of SICN NPs Stabilized Particle Sizes}

The SICN nanoparticles prepared by a simple precipitation method have good water solubility and are easily soluble in water and acid water. The measured particle sizes of SICN in water and acid water are $61.5 \pm 0.22 \mathrm{~nm}$ and 50 $\pm 0.24 \mathrm{~nm}$, respectively (Figure S1). It can be seen that there is little difference in the particle size of SICN in different acid-base solutions, and it is reasonable to infer that SICN nanoparticles are not easily affected by the acidic environment in solid tumors. 


\section{Morphology and Appearance}

The appearance and shape of SICN were observed under scanning electron microscope (SEM), as shown in Figure S2. SICN showed the shape of spherical particles, which exist individually or aggregated together. The results of transmission electron microscope (TEM) also showed that SICN was spherical nanoparticles (Figure S3), which was consistent with the results of SEM.

\section{XRD Analysis}

The XRD analysis of SICN and its components is shown in Figure S4 (scanning range of 5-50॰). Compared with irinotecan and curcumin, SICN has new diffraction peaks in many places, which means that a new crystal is formed.

\section{The Drug Release and Fluorescence Change}

The fluorescence change of SICN in the aqueous solution is shown in Figure S5B. In general, the fluorescence gradually increased within 36 hours and then decreased. There is almost no difference in the maximum fluorescence value in the range of 4-24h ( $p>0.05$ ). According to the fluorescence emission curve, the maximum emission wavelength of irinotecan is $440 \mathrm{~nm}$, and the maximum emission wavelength of curcumin is $540 \mathrm{~nm}$ (Figure S5A). We believe that the change in fluorescence at $440 \mathrm{~nm}$ is caused by the release of irinotecan. It can be seen that the release of irinotecan gradually increased within 36h (no significant difference between 4 24h $(P>0.05)$, then the drug release slowed down and the fluorescence began to weaken due to the fluorescence quenching effect. The release and fluorescence changes of SICN in PBS solution are consistent with those in aqueous solution (Figure S5C). It shows that SICN exhibits the same drug release and fluorescence change behavior in different solvents. The difference is that the release in water is faster than in PBS solution (the fluorescence intensity of SICN in aqueous solution is always higher than in PBS solution).

\section{Uptake and Release of SICN in Cells}

Flow cytometry studies compared the uptake and release of SICN nanoparticles and free curcumin. As shown in Figure $2 \mathrm{~A}$ and $\mathrm{B}$, the control experiment in HGC-27 cells incubated without SICN/curcumin showed a much weaker fluorescence signal. In the first $4 \mathrm{~h}$, the fluorescence of SICN nanoparticles and curcumin increased with incubation time, indicating that both SICN nanoparticles and curcumin can be absorbed by HGC-27 cells. Moreover, the uptake of SICN nanoparticles was significantly higher than free curcumin. Quantitative data in flow cytometry analysis are shown in Figure 2E, exhibiting that the fluorescence signals of SICN and free curcumin are weakened after $4 \mathrm{~h}$. This may be due to the drug being released in or discharged from the cell. SICN fluorescence signals decreased steadily and slowly within $48 \mathrm{~h}$, whereas free curcumin continuously decreased significantly, which is one reason for the low bioavailability of curcumin. Therefore, SICN nanoparticles are released and discharged slowly and steadily in the cell. It means that the nanoplatform can control the release of drugs, improve bioavailability, help achieve long-term efficacy, and reduce the number of administrations.

To further compare the two drugs' intracellular excretion rate, flow cytometry was used to monitor the fluorescence intensity of SICN/curcumin after co-incubation with cells in real-time (after $4 \mathrm{~h}$ co-incubation, the drugcontaining medium was replaced with fresh medium and marked as $0 \mathrm{~h}$ ). As shown in Figure $2 \mathrm{C}$ and D, the excretion rate of free curcumin was significantly higher than SICN, and it was continuously excreted, whereas SICN excretion was slower between $8 \mathrm{~h}$ and $24 \mathrm{~h}$. Quantitative data in flow cytometry analysis (Figure $2 \mathrm{~F}$ ) showed the same consequence. It also showed that the spontaneous fluorescence of SICN could realize real-time monitoring at the cellular level.

\section{Uptake and Release of SICN in Zebrafish}

Zebrafish were chosen as an in vivo model for optical monitoring due to the model's convenient optical imaging. Based on the spontaneous fluorescence of curcumin, SICN nanoparticles can also be self-monitored. This feature can monitor the uptake of SICN and curcumin in zebrafish in real-time. Zebrafish embryos were simply fed with curcu$\mathrm{min} / \mathrm{SICN}$ and monitored using fluorescence imaging for $72 \mathrm{~h}$. As shown in Figures 3A, B and S7A, SICN and curcumin can be rapidly absorbed within $2 \mathrm{~h}$ by zebrafish, and then SICN was rapidly distributed in the whole body. In the first $4 \mathrm{~h}$, SICN was absorbed in the liver and gastrointestinal tract. After $4 \mathrm{~h}$, SICN was distributed in the whole body. As the drug was released and metabolized for a long time in vivo, its fluorescence could still be monitored until $72 \mathrm{~h}$. In contrast, curcumin was only distributed in the gastrointestinal tract up to $4 \mathrm{~h}$ after being taken in and then distributed to the whole body and was gradually metabolized out of the body.

Real-time uptake experiments suggested that SICN can penetrate the tissue of a live zebrafish with ease. The death of zebrafish could not be observed in the effective dose of 



E
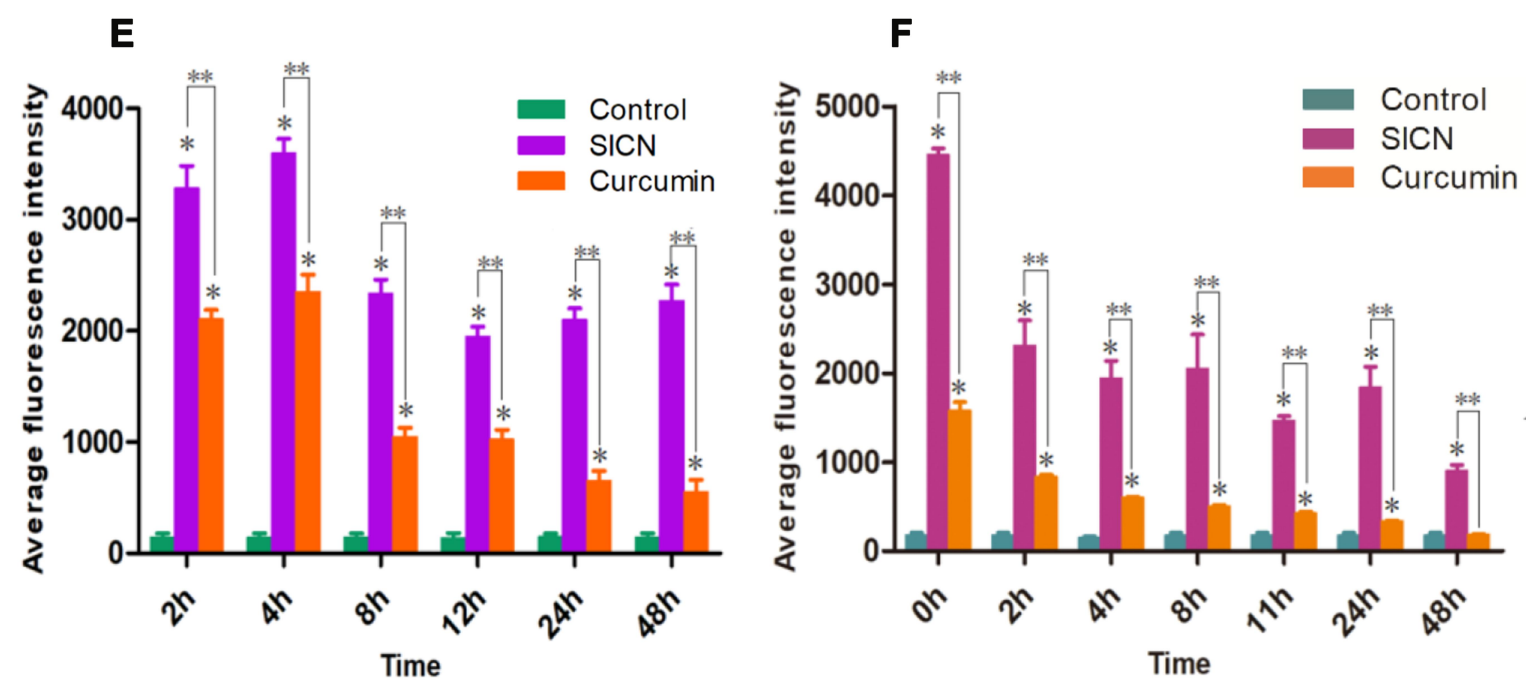

Figure 2 In vitro real-time self-monitoring uptake and release of drugs. (A and B) In vitro flow cytometric analysis of fluorescent intensities of SICN (A) and curcumin (B) in HGC-27 cells at designated time intervals, it represents the uptake of SICN and curcumin. (C and D) In vitro flow cytometric analysis of fluorescent intensities in HGC-27 cells at designated time intervals after $\operatorname{SICN}(\mathbf{C})$ and curcumin (D) were removed, it represents the excretion of SICN and curcumin. (E and $\mathbf{F}$ ) Fluorescence quantitative data of SICN and curcumin in HGC-27 cells at designated time intervals (E for uptake and F for excretion). Data represent the mean \pm SD, *Represents significant difference compared with control group $(P<0.05)$, **Represents significant difference between irinotecan group and curcumin group $(P<0.05)$. 


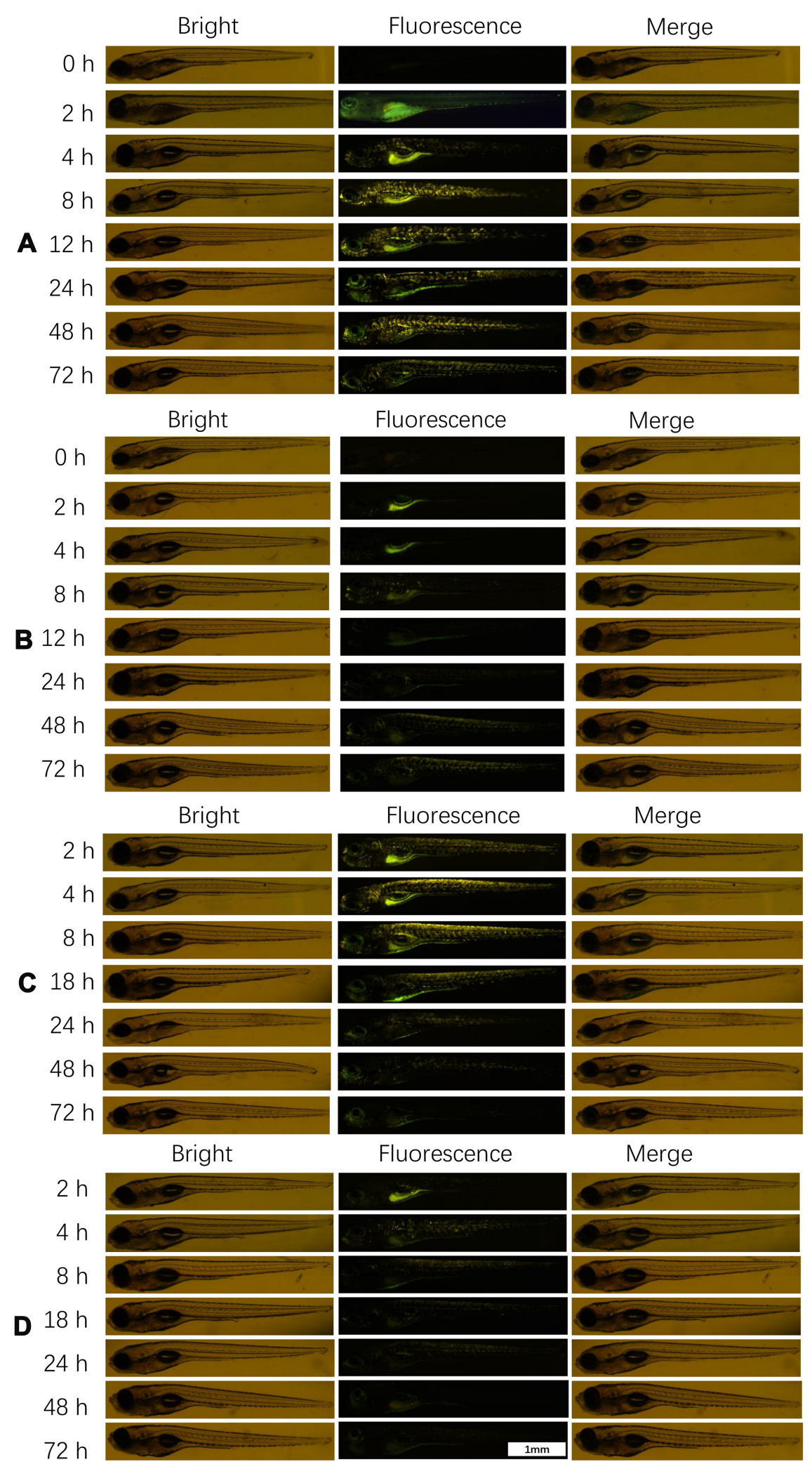

Figure 3 In vivo imaging of zebrafish uptake and release of SICN and curcumin. (A and B) In vivo imaging of fluorescent intensities of SICN (A) and curcumin (B) in zebrafish at designated time intervals. (C and D) In vivo imaging of fluorescent intensities in zebrafish at designated time intervals after SICN (C) and curcumin (D) were removed. 
SICN. Therefore, it was boldly speculated that SICN might have the ability to penetrate the tissue at the whole organism level or even spread to all tissue regions without causing life-threatening side effects. Previous studies have also shown that SICN did not damage the main organs, ${ }^{23}$ indicating that SICN can expand the scope of application and play a more extensive antitumor effect.

Similar to in vitro experiments, to monitor drug excretion in the body, zebrafish were incubated with drugs for 4 $\mathrm{h}$ and then cultured in fresh fish-water for observation. SICN was shown to be relatively stable and distributed throughout the zebrafish body within $8 \mathrm{~h}$. Even at $18 \mathrm{~h}$, there was a significant drug fluorescence distribution, and it was then gradually metabolized and excreted with time (Figure 3C). Conversely, curcumin stayed in the body for a short time and was metabolized and excreted quickly after the administration was stopped (Figures 3D and S7B). It has been proven that the longer the half-life of nanomedicine in the blood circulation is, the longer is the accumulation time of drugs in tumor tissues, which is conducive to enhance the role of enhanced permeability and retention (EPR) and reduce the frequency of taking drugs. ${ }^{27}$ Due to the long circulation of SICN in vivo, it has the potential to enhance the EPR effect.

\section{Endocytosis and Intracellular Transport of SICN Nanoparticles}

The frequently used method to determine the endocytic pathways of nanosystems is the use of pharmacological inhibitors. $^{28,29}$ To track the internalization pathways of SICN nanoparticles, confocal microscopy is used to capture the fluorescence of SICN nanoparticles in cells pretreated with small-molecule endocytosis inhibitors. As shown in Figure 4A and B, the cellular uptake of SICN was significantly blocked at $4{ }^{\circ} \mathrm{C}$, suggesting an energy-dependent internalization. Further, cellular uptake was significantly inhibited by cytochalasin $D$, methyl- $\beta$-cyclodextrin, wortmannin, and genistein (Figure 4C and D). Cytochalasin D and wortmannin are macropinocytosis inhibitors, genistein is a caveolin

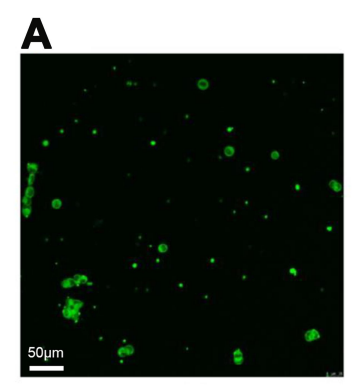

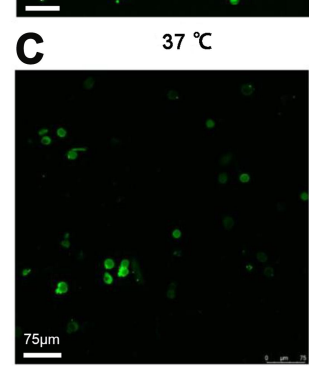

Without inhibitor

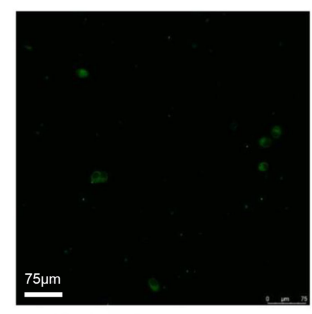

Methyl- $\beta$-cyclodextrin

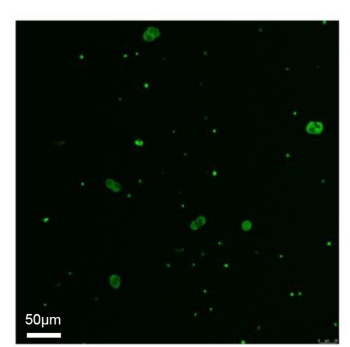

$4{ }^{\circ} \mathrm{C}$

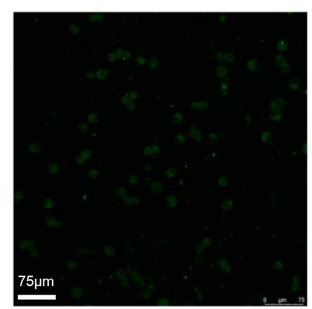

Cytochalasin D

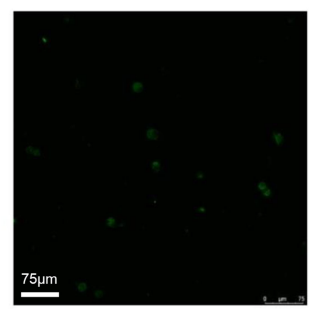

Wortmannin
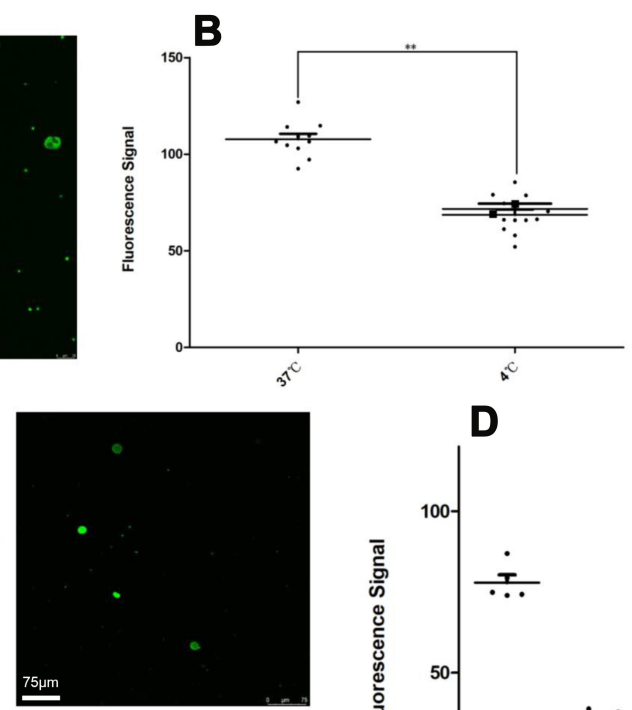

Chlorpromazine

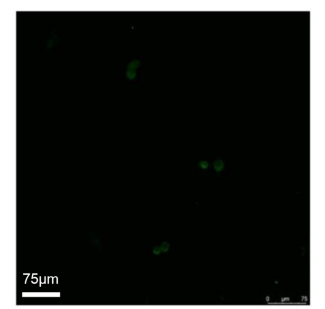

Genistein

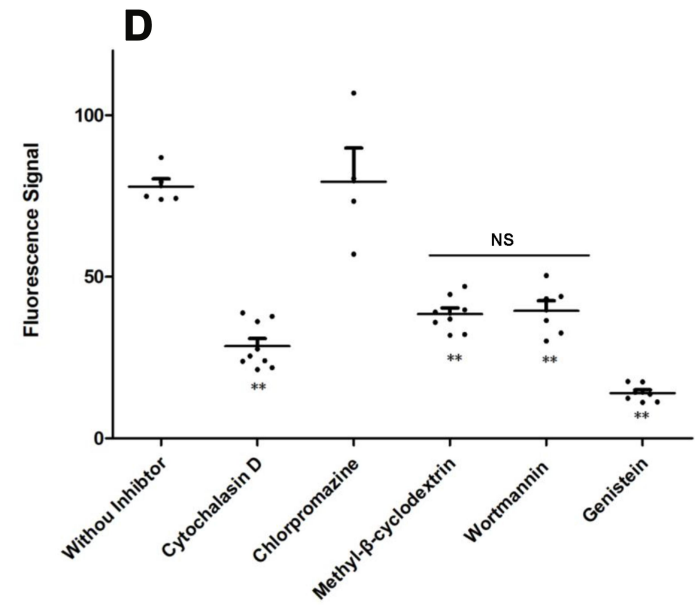

Figure 4 Fluorescence-based effects of inhibitors on the endocytosis of SICN nanoparticles on $\mathrm{HGC}-27$ cells. (A) Cellular uptake of SICN nanoparticles at $37^{\circ} \mathrm{C}$ and $4^{\circ} \mathrm{C}$ environments as assessed by CLSM. (B) Data are mean \pm standard deviation (SD). ${ }^{* *} p<0.00 \mathrm{I}$, significant difference between normal and $4^{\circ} \mathrm{C}$ groups. (C) Cellular uptake of SICN nanoparticles in the absence ("without inhibitor" control) and presence of inhibitors, as assessed by CSLM. (D) Data are mean \pm SD. **p < 0.00 I, significant difference between control and inhibitor groups.

Abbreviation: NS, no statistical difference. 
inhibitor, and methyl- $\beta$-cyclodextrin simultaneously affects clathrin-mediated and independent endocytosis, indicating that SICN is internalized into gastric cancer cells through multiple pathways.

To further explore the distribution of SICN in cells after internalization, cells were stained with lysosomes and Golgi apparatus markers. As shown in Figure 5A and $\mathrm{B}$, besides colocalization with lysosomes and Golgi apparatus, the fluorescence signal of SICN is almost distributed in the whole cytoplasm. Nanoparticles transported to lysosomes are easily metabolically inactivated, but most SICN can be delivered out of the cell in the form of original drugs or active ingredients through the Golgi apparatus and other channels and continuously act on the next cell, with the potential to penetrate deep into the tumor.

\section{Cell Cytotoxicity}

Human gastric cancer cells (HGC-27) were utilized to assess the cytotoxicity of irinotecan hydrochloride, $\mathrm{SICN}$, curcumin. As shown in Figure $6 \mathrm{~A}$, the $\mathrm{IC}_{50}$ value (half-maximal inhibitory concentration) of SICN nanoparticles against $\mathrm{HGC}-27$ cells was $0.151 \mu \mathrm{M} / \mathrm{L}$ at the irinotecan hydrochloride equivalent and $0.481 \mu \mathrm{M} /$
L in irinotecan hydrochloride. Cytotoxicity of SICN nanoparticles against HGC-27 cells was slightly higher than irinotecan hydrochloride, with a significant difference $(p<0.05)$. Previous studies have confirmed that the conversional positive surface charges of SICN nanoparticles under acidic tumor environments and the negative surface charges under normal physiological conditions make the acidic environment more likely to cause in vitro cytotoxicity than the alkaline environment. $^{23}$ As shown in Figure 6C, the uptake of SICN by HGC-27 cells in a weak acid environment ( $\mathrm{pH}$ 6.7) was increased by $68.5 \%$ compared to a weak alkaline environment ( $\mathrm{pH}$ 7.5), and SICN showed stronger cytotoxicity to HGC-27 cells in a weak acid environment (Figure 6B). Due to the tunability of the surface charge of nanoparticles, it was more conducive for absorption by tumor cells in a weakly acidic tumor environment.

In order to provide more evidence for the cytotoxicity of SICN, we also used A2780 human ovarian cancer cells and A549 human non-small cell lung cancer cells to study the cytotoxicity of SICN (Figure S5). Similar to HGC-27 cells, SICN shows strong cytotoxicity to A2780 cells and moderate cytotoxicity to A549 cells.
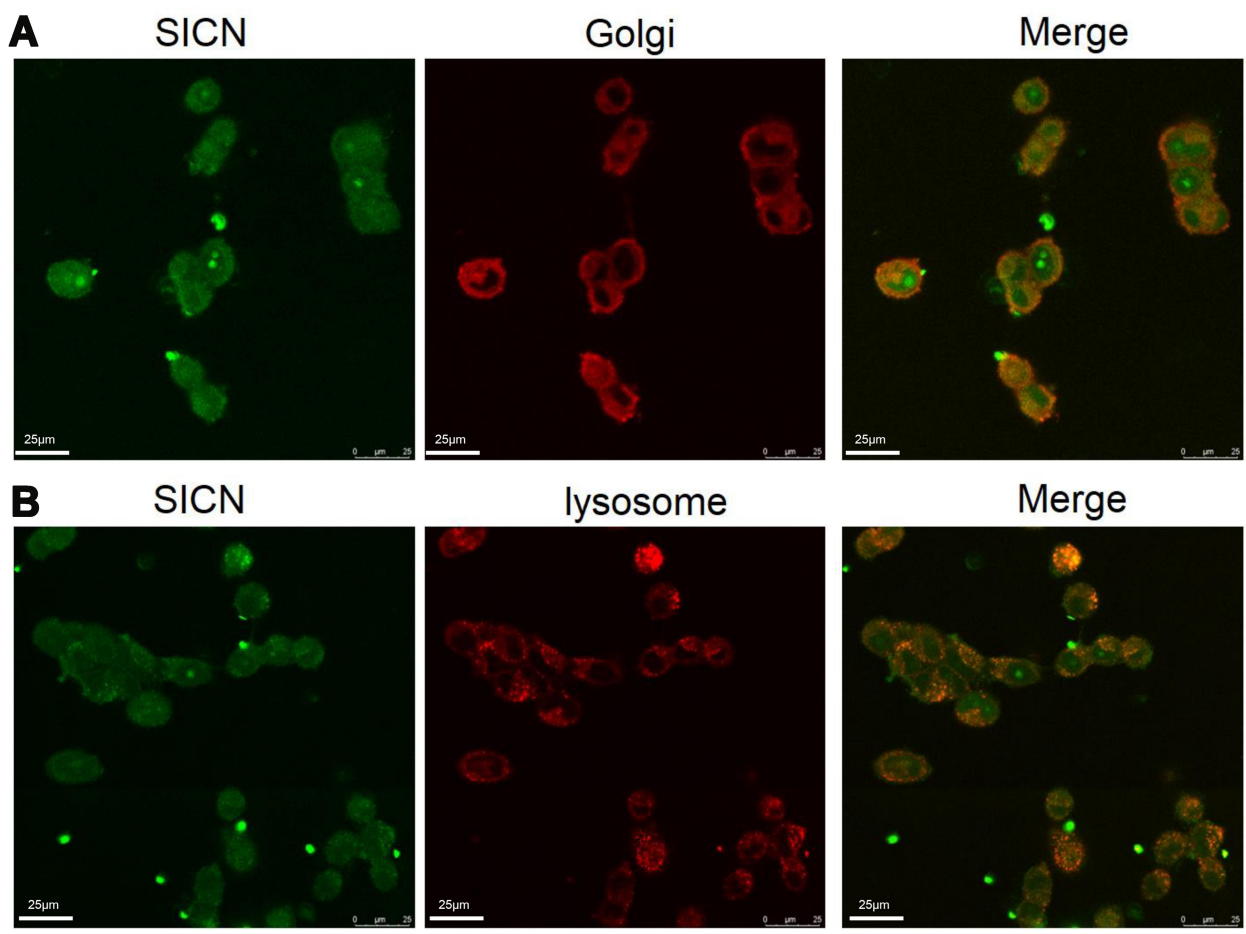

Figure 5 Intracellular trafficking of SICN in HGC-27 cells. Colocalization of SICN and Golgi-Tracker Red (A) or LysoTracker Red (B) after 6 h culture with HGC-27 cells. 

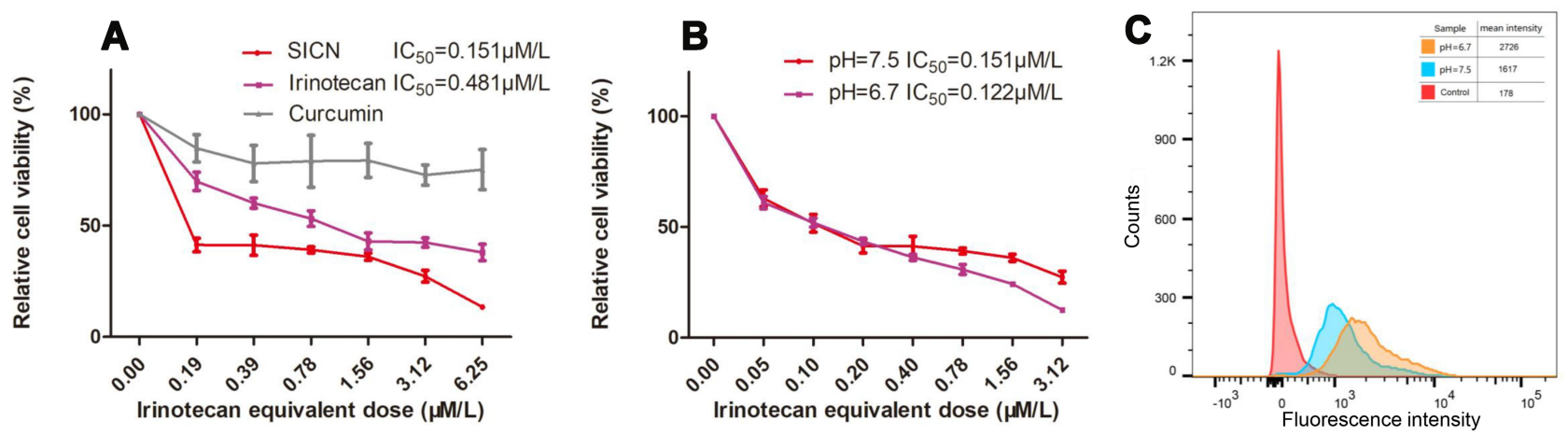

Figure 6 Cell cytotoxicity assays of irinotecan hydrochloride and SICN nanoparticles. (A) MTT assay curves of SICN, irinotecan hydrochloride and curcumin against HGC27 cells for $48 \mathrm{~h}$ treatment ( $\mathrm{n}=3$ independent experiments using the same batch of drugs). (B) MTT assay curves of SICN by changing the environmental $\mathrm{PH}$ values on $\mathrm{HGC}$ 27 cells. (C) The fluorescence intensity of SICN in A2780 cells detected by flow cytometry in different pH environments.

\section{Cell Apoptosis}

To investigate the cell death mechanism of SICN, HGC-27 cells were double-labeled with Annexin V-FITC and PI before analysis by flow cytometry. Cell populations at different phases of cell death, namely, live (Q4), early apoptotic (Q3), late-stage apoptotic (Q2), and necrotic (Q1), at different treatments are shown in the Figure 7. Compared to the control group (Figure 7A), there was no significant difference between the cell populations in the curcumin group (Figure 7B). In contrast, the percentage of total apoptotic cells (including early and late apoptosis) of the irinotecan hydrochloride (Figure 7C) and SICN nanoparticles groups (Figure 7D) dramatically increased to $35.6 \%$ and $60.2 \%$, respectively, which were much more than the control group

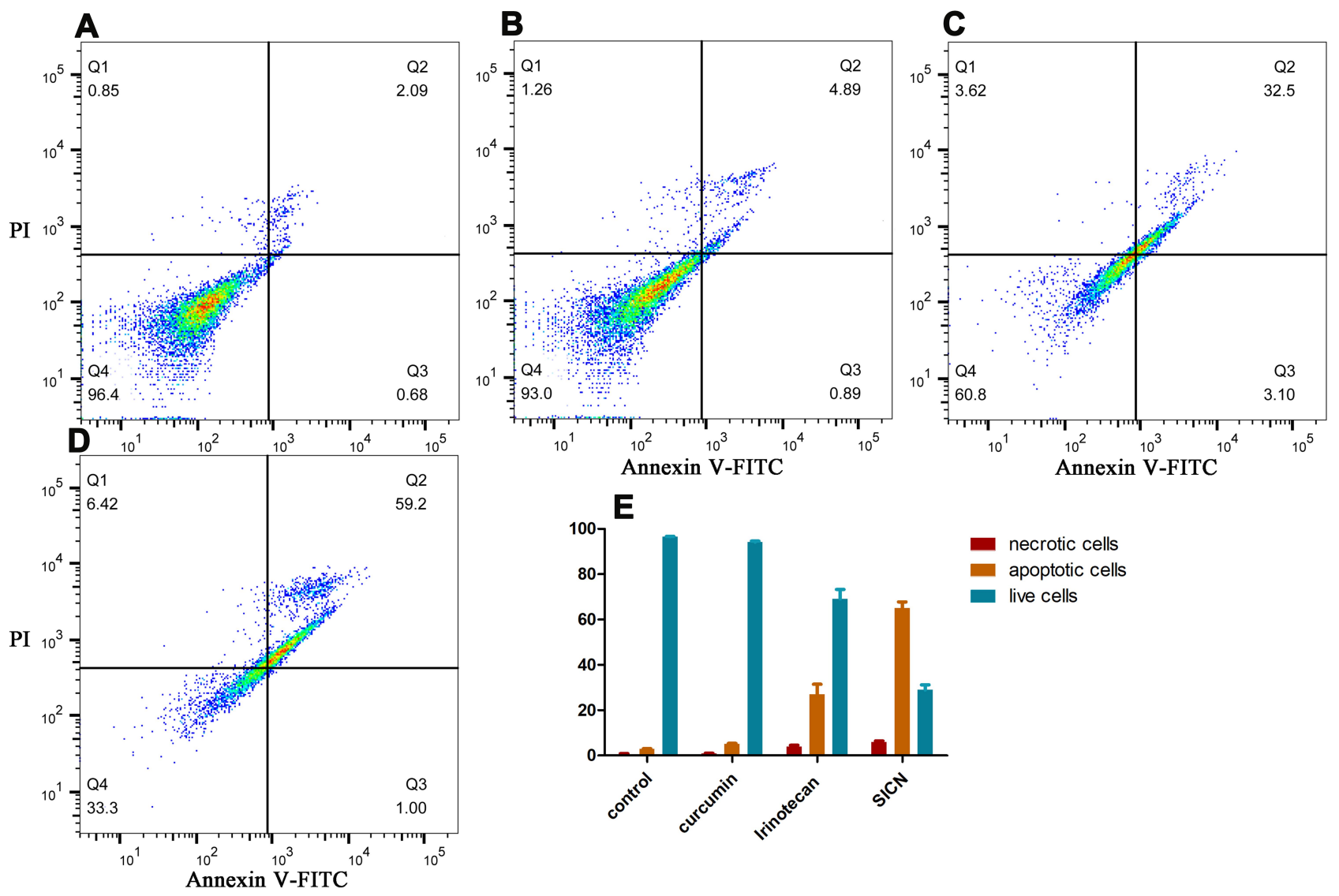

Figure 7 Apoptosis of HGC-27 cells induced by curcumin, irinotecan hydrochloride or SICN nanoparticles. (A) Control group, without treatment; (B) curcumin group; (C) irinotecan hydrochloride group; (D) SICN nanoparticles group; and (E) percentage of cell populations at different phases of the cell death. 

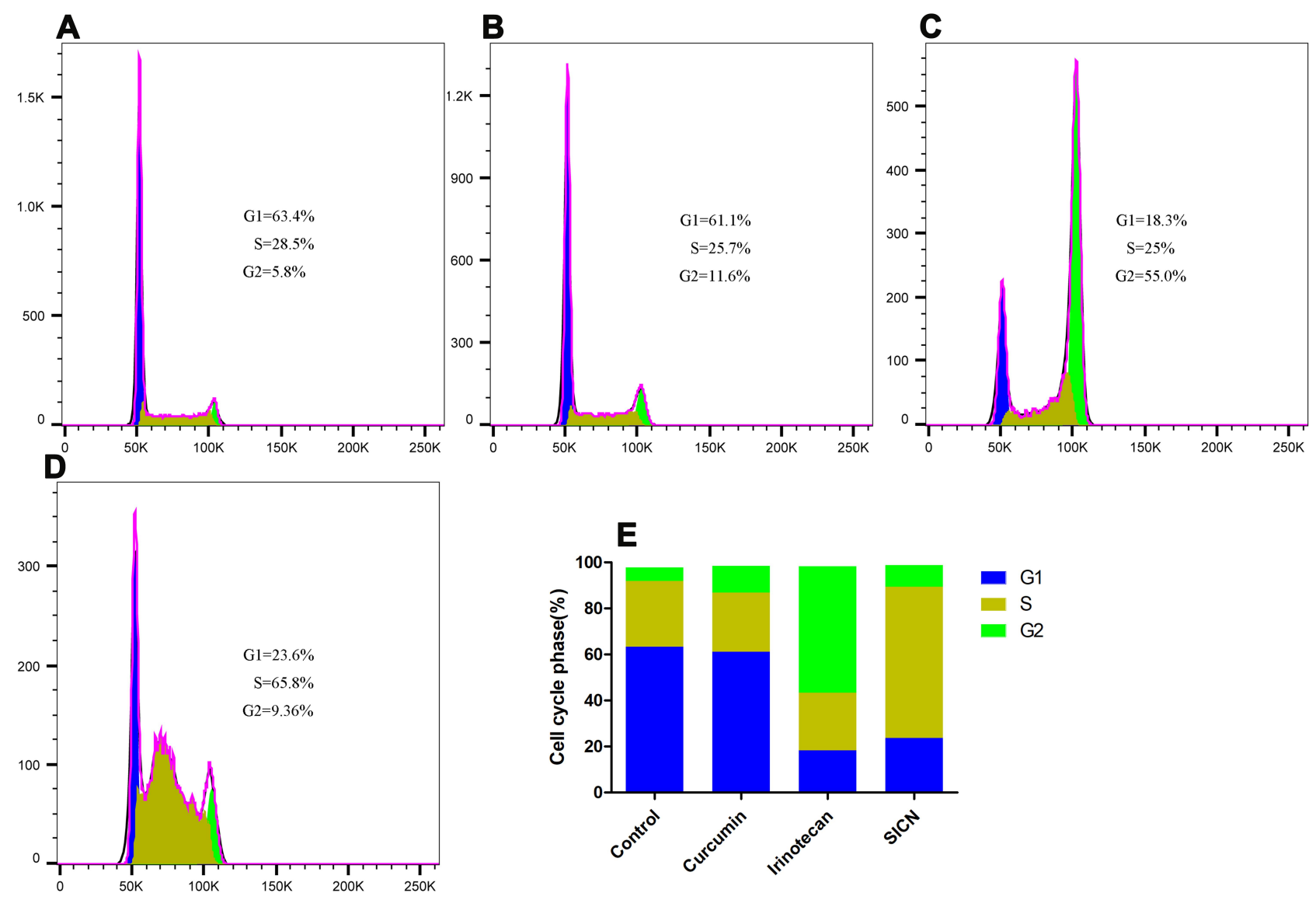

Figure 8 Cell cycle of HGC-27 cells induced by curcumin, irinotecan hydrochloride, or SICN nanoparticles. (A) Control group, without treatment; (B) curcumin group; (C) irinotecan hydrochloride group; (D) SICN nanoparticles group; and (E) percentage of cell populations at different phases of the cell cycle.

(2.77\%). Both drugs caused late-stage apoptosis. The percentages of necrotic cells increased slightly to $3.62 \%$ and $6.42 \%$, which were higher than the control group $(0.85 \%)$. More apoptotic and necrotic cells could be observed when cells were treated with SICN (Figure 7E). These results suggested that the designed nanoparticles were potential and effective strategy for apoptosis of HGC-27 cells.

\section{Cell Cycle}

PI was used to measure the cell DNA content. As shown in Figure $8 \mathrm{~A}$ and B, treatment of HGC-27 cells with curcumin did not alter the cell cycle compared to the control group. Cells treated with irinotecan hydrochloride showed $55.0 \%$ arrest in the $\mathrm{G}_{2}$ phase of the cell cycle, leading to cell death (Figure 8C), compared to $5.8 \%$ in the control group. Nevertheless, cells treated with SICN exhibited a different block phase, which was arrest in the S phase at $65.8 \%$ (Figure $8 \mathrm{D}$ ) compared to $28.5 \%$ in the control group. It can be clearly seen that SICN nanoparticles caused DNA damage by blocking the S phase of DNA replication in HGC-27 cells (Figure 8E).

\section{Conclusion}

In summary, based on the established carrier-free nanosystems of curcumin-irinotecan hydrochloride, the multifunctional strategy of this anticancer combination therapy was further studied. Based on the fluorescence of curcumin, flow cytometry, zebrafish fluorescence imaging, and confocal microscopy imaging indicated that SICN nanoparticles could be used as a real-time selfmonitoring platform. It can monitor the uptake, absorption and excretion of nanoparticles in vivo and in vitro in real time. Meanwhile, it can also be used to study the internalization and intracellular transfer pathways of nanoparticles. Cytotoxicity experiments have shown that combination therapy leads to higher toxicity than single-component chemotherapy. Because nanoparticles can switch charge in a slightly acidic tumor environment, HGC-27 cells have more uptake of nanoparticles and higher toxicity under slightly acidic conditions. Overall, this multifunctional therapeutic strategy based on the carrier-free autofluorescent drug curcumin and irinotecan 
hydrochloride to construct nanoparticles may open new ideas for combined cancer treatment and monitoring.

\section{Acknowledgments}

We express our great appreciation to Jiayi Sun at Innovative Institute of Chinese Medicine and Pharmacy, Chengdu University of Traditional Chinese Medicine, for the assistance with confocal laser scanning microscopy. This research was supported by National Natural Science Foundation of China Youth Science Fund Project (81903777), Ministry of Education, Youth and Sports of the Czech Republic (RP/ CPS/2020/002), Sichuan Province Science and Technology Basic Condition Platform Project (2019JDPT0032), Xinglin Scholars Discipline Talent Research Promotion Plan (CXTD2018012) and the Open Research Fund of Chengdu University of Traditional Chinese Medicine Key Laboratory of Systematic Research of Distinctive Chinese Medicine Resources in Southwest China (2020XSGG014).

\section{Disclosure}

The authors report no conflicts of interest in this work.

\section{References}

1. Siegel RL, Miller KD, Jemal A. Cancer statistics, 2020. CA Cancer J Clin. 2020;70(1):7-30. doi:10.3322/caac.21590

2. Balakumar P, Maung UK, Jagadeesh G. Prevalence and prevention of cardiovascular disease and diabetes mellitus. Pharmacol Res. 2016;113:600-609. doi:10.1016/j.phrs.2016.09.040

3. Miller KD, Siegel RL, Lin CC, et al. Cancer treatment and survivorship statistics, 2016. CA Cancer J Clin. 2016;66(4):271-289. doi:10.3322/caac. 21349

4. Wang N, Zhou Y, Xu Y, et al. Molecular engineering of anti-PD-L1 peptide and photosensitizer for immune checkpoint blockade photodynamic-immunotherapy. Chem Eng J. 2020;400:125995. doi:10.1016/j.cej.2020.125995

5. Keall P, Nguyen DT, O'Brien R, et al. Real-time image guided ablative prostate cancer radiation therapy: results from the TROG 15.01 SPARK Trial. Int J Radiat Oncol Biol Phys. 2020;107 (3):530-538. doi:10.1016/j.ijrobp.2020.03.014

6. Shaw AT, Kim DW, Nakagawa K, et al. Crizotinib versus chemotherapy in advanced ALK-positive lung cancer. $N$ Engl J Med. 2013;368:2385-2394. doi:10.1056/NEJMoa1214886

7. Van Driel WJ, Koole SN, Sikorska K, et al. Hyperthermic intraperitoneal chemotherapy in ovarian cancer. $N$ Engl J Med. 2018;378:230-240. doi:10.1056/NEJMoa1708618

8. Bertrand N, Wu J, Xu X, et al. Cancer nanotechnology: the impact of passive and active targeting in the era of modern cancer biology. Adv Drug Deliv Rev. 2014;66:2-25. doi:10.1016/j.addr.2013.11.009

9. Pham DT, Chokamonsirikun A, Phattaravorakarn V, et al. Polymeric micelles for pulmonary drug delivery: a comprehensive review. J Mater Sci. 2020;56:2016-2036. doi:10.1007/s10853-020-05361-4

10. Mirzaie Z, Barati M, Tokmedash MA. Anticancer drug delivery systems based on curcumin nanostructures: a review. Pharm Chem J. 2020;54(4):353-360. doi:10.1007/s11094-020-02203-0

11. Cai AY, Zhu YJ, Qi C. Biodegradable inorganic nanostructured biomaterials for drug delivery. Adv Mater Interfaces. 2020;7 (20):1-30. doi:10.1002/admi.202000819
12. Perez-Herrero E, Fernandez-Medarde A. Advanced targeted therapies in cancer: drug nanocarriers, the future of chemotherapy. Eur J Pharm Biopharm. 2015;93:52-79. doi:10.1016/j.ejpb.2015.03.018

13. Zhou Q, Shao S, Wang J, et al. Enzyme-activatable polymer-drug conjugate augments tumour penetration and treatment efficacy. Nat Nanotechnol. 2019;14(8):799-809. doi:10.1038/s41565-019-0485-Z

14. Zhang J, Liang Y, Lin X, et al. Self-monitoring and self-delivery of photosensitizer-doped nanoparticles for highly effective combination cancer therapy in vitro and in vivo. ACS Nano. 2015;9 (10):9741-9756. doi:10.1021/acsnano.5b02513

15. Kanamala M, Wilson WR, Yang M, et al. Mechanisms and biomaterials in $\mathrm{pH}$-responsive tumour targeted drug delivery: a review. Biomaterials. 2016;85:152-167. doi:10.1016/j.biomaterials.2016.01.061

16. Jia X, Zhang Y, Zou Y, et al. Dual intratumoral redox/enzyme-responsive NO-releasing nanomedicine for the specific, high-efficacy, and low-toxic cancer therapy. Adv Mater. 2018;30:1-9. doi:10.1002/ adma.201704490

17. Zhang Y, Hu H, Tang W, et al. A multifunctional magnetic nanosystem based on "two strikes" effect for synergistic anticancer therapy in triple-negative breast cancer. J Control Release. 2020;322:401-415. doi:10.1016/j.jconrel.2020.03.036

18. Zhang Y, Yan J, Avellan A, et al. Temperature and $\mathrm{pH}$ responsive star polymers as nano-carriers with potential for in vivo agrochemical delivery. ACS Nano. 2020. doi:10.1021/acsnano.0c03140

19. Unnikrishnan BS, Maya S, Preethi GU, et al. Self-assembled drug loaded glycosyl-protein metal nanoconstruct: detailed synthetic procedure and therapeutic effect in solid tumor treatment. Colloids Surf $B \quad$ Biointerfaces. 2020;193. doi:10.1016/j.colsurfb. 202 0.111082

20. Zhang ZT, Wang RY, Huang XX, et al. Self-delivered and self-monitored chemo-photodynamic nanoparticles with light-triggered synergistic antitumor therapies by downregulation of HIF-1 alpha and Depletion of GSH. ACS Appl Mater Interfaces. 2020;12(5):5680-5694. doi:10.1021/acsami.9b23325

21. Parisi OI, Ruffo M, Malivindi R, et al. Molecularly imprinted polymers (MIPs) as theranostic systems for sunitinib controlled release and self-monitoring in cancer therapy. Pharmaceutics. 2020;12 (1):1-18. doi:10.3390/pharmaceutics12010041

22. Zhang H, Fan T, Chen W, et al. Recent advances of two-dimensional materials in smart drug delivery nano-systems. Bioact Mater. 2020;5 (4):1071-1086. doi:10.1016/j.bioactmat.2020.06.012

23. Xiao H, Guo Y, Liu $H$, et al. Structure-based design of charge-conversional drug self-delivery systems for better targeted cancer therapy. Biomaterials. 2020;232. doi:10.1016/j. biomaterials.2019.119701

24. Hassanzadeh K, Buccarello L, Dragotto J, et al. Obstacles against the marketing of curcumin as a drug. Int J Mol Sci. 2020;21(18):1-35. doi:10.3390/ijms21186619

25. Scazzocchio B, Minghetti L, D'Archivio M. Interaction between gut microbiota and curcumin: a new key of understanding for the health effects of curcumin. Nutrients. 2020;12(9):1-18. doi:10.3390/ nu12092499

26. Westerfield M. The Zebrafish Book. A Guide for the Laboratory Use of Zebrafish (Danio Rerio). 4th ed. Eugene: University of Oregon Press; 2000.

27. Fang J, Islam W, Maeda H. Exploiting the dynamics of the EPR effect and strategies to improve the therapeutic effects of nanomedicines by using EPR effect enhancers. Adv Drug Deliv Rev. 2020;157:142-160. doi:10.1016/j.addr.2020.06.005

28. Chen K, Li X, Zhu H, et al. Endocytosis of nanoscale systems for cancer treatments. Curr Med Chem. 2018;25(25):3017-3035. doi:10.2174/0929867324666170428153056

29. Iversen T-G, Skotland T, Sandvig K. Endocytosis and intracellular transport of nanoparticles: present knowledge and need for future studies. Nano Today. 2011;6(2):176-185. doi:10.1016/j. nantod.2011.02.003 


\section{Publish your work in this journal}

The International Journal of Nanomedicine is an international, peerreviewed journal focusing on the application of nanotechnology in diagnostics, therapeutics, and drug delivery systems throughout the biomedical field. This journal is indexed on PubMed Central, MedLine, CAS, SciSearch ${ }^{\mathbb{B}}$, Current Contents ${ }^{\mathbb{B}} /$ Clinical Medicine,
Journal Citation Reports/Science Edition, EMBase, Scopus and the Elsevier Bibliographic databases. The manuscript management system is completely online and includes a very quick and fair peer-review system, which is all easy to use. Visit http://www.dovepress.com/ testimonials.php to read real quotes from published authors.

Submit your manuscript here: https://www.dovepress.com/international-journal-of-nanomedicine-journal 